\title{
Coming of age in L3 initial stages transfer models: deriving developmental predictions and looking towards the future
}

Article

Accepted Version

Gonzalez Alonso, J. and Rothman, J. (2017) Coming of age in L3 initial stages transfer models: deriving developmental predictions and looking towards the future. International Journal of Bilingualism, 21 (6). pp. 683-697. ISSN 1756-6878 doi: https://doi.org/10.1177/1367006916649265 Available at https://centaur.reading.ac.uk/61776/

It is advisable to refer to the publisher's version if you intend to cite from the work. See Guidance on citing.

To link to this article DOI: http://dx.doi.org/10.1177/1367006916649265

Publisher: Sage

All outputs in CentAUR are protected by Intellectual Property Rights law, including copyright law. Copyright and IPR is retained by the creators or other copyright holders. Terms and conditions for use of this material are defined in the End User Agreement.

www.reading.ac.uk/centaur 
Central Archive at the University of Reading

Reading's research outputs online 


\title{
Coming of age in L3 initial stages transfer models: Deriving developmental predictions and looking towards the future
}

\author{
Jorge González Alonso ${ }^{1,2}$ and Jason Rothman ${ }^{1,2}$ \\ 1. University of Reading 2. UiT The Arctic University of Norway
}

\section{Abstract}

Aims: Over the past decade in particular, formal linguistic work within L3 acquisition has concentrated on hypothesizing and empirically determining the source of transfer from previous languages - L1, L2 or both-in L3 grammatical representations. In view of the progressive concern with more advanced stages, we aim to show that focusing on L3 initial stages should be one continued priority of the field, even —or especially—if the field is ready to shift towards modeling L3 development and ultimate attainment.

Approach: We argue that L3 learnability is significantly impacted by initial stages transfer, as such forms the basis of the initial L3 interlanguage. To illustrate our point, the insights from studies using initial and intermediary stages L3 data are discussed in light of developmental predictions that derive from the initial stages models.

Conclusions: Despite a shared desire to understand the process of L3 acquisition in whole, inclusive of offering developmental L3 theories, we argue that the field does not yet havealthough is ever closer to- the data basis needed to effectively do so. 
Originality: This article seeks to convince the readership for the need of conservatism in L3 acquisition theory building, whereby offering a framework on how and why we can most effectively build on the accumulated knowledge of the L3 initial stages in order to make significant, steady progress.

Significance: The arguments exposed here are meant to provide an epistemological base for a tenable framework of formal approaches to L3 interlanguage development and, eventually, ultimate attainment.

\section{Keywords}

L3 acquisition, learnability, Subset Principle, initial stages, developmental predictions. 


\section{General introduction}

It is relatively uncontroversial to assume that previous linguistic knowledge affects the process of subsequent language acquisition, known in the literature as cross-linguistic influence (CLI) and/or transfer, depending on its nature. Third language (L3) learners have a greater number of linguistic representations available to them at the start of L3 acquisition (L3A) and throughout as compared to second language (L2) learners. These co-existing L1 and L2 representations have different mapping combinations with respect to underlying grammatical properties, as they can (i) encode the same property similarly, (ii) embody two different configurations of the same property, or (iii) be the expression of grammatical properties that are not realized in the other language. Because a beginning L2 learner (L2er) only has access to one system, to the extent that transfer obtains, it is relatively easy to determine what the source has to be. In L3/Ln acquisition, with multiple potential resource grammars, determining the transfer source(s) is not as straightforward as the default case in L2 acquisition (L2A). In addition to the larger complexity of the L3 initial state, the possibilities expressed in (i-iii) have important implications for L3 learnability, that is, constraints on developmental sequence and even ultimate attainment. Herein, we argue that how these possibilities play out in the L3 initial stages is ultimately one deterministic variable that feeds into L3 learnability. Prioritizing the start of the L3 process, as many studies have done, is thus not surprising since only by fully understanding the onset of any process do we have the best chance of ultimately revealing the nature of the rest. Of course, an accurate characterization of the beginning does not necessarily reveal or predict the dynamics of the whole, but understanding the initial point of any given 
process increases our chances of success when attempting to model it in its entirety.

Having acknowledged the crucial importance of understanding the dynamics of the initial stages of L3A, we concede that the emerging field of formal approaches to L3 morphosyntax needs to engage with the entire process of L3A more directly. In due course, there will be specific models of L3 development and ultimate attainment. At present there are the beginnings of such proposals, with two notable examples in Slabakova's (2015) Scalpel Model and Mykhaylyk et al.'s (2015) Linguistic Proximity Model. These models embody a call, indicating that the field is ready for a shift in focus towards understanding L3A more completely. While we will argue that the field is not quite ready for comprehensive theories of L3 development, we do believe that the past decade's accumulated knowledge on the nature of the initial stages of L3A has prepared us to work towards this goal. Our specific goal herein is thus twofold: (i) to elucidate the importance of first understanding the mental representations of the initial L3 interlanguage grammar(s), inclusive of determining the source(s) of transfer that form its basis, and (ii) building on (i), to show how we can conservatively expand the basis of existent initial stages models of morphosyntax into L3 development within an extended framework that makes predictions and is thus falsifiable.

\section{Models of transfer in L3 morphosyntax}

As is the case for all initial stages of language learning irrespective of age and experience (L1, 2L1, child and adult L2), L3ers are faced with the challenging task of making sense of a large amount of input. The L3 situation is perhaps most comparable to that of L2A (and different from L1A) in that the learner has previous linguistic experience to draw from, and so 
transferring some or all of this knowledge may change the way in which the learning task in undertaken. At the same time, the $\mathrm{L} 3 / \mathrm{Ln}$ setting is unique in that the learner's previous linguistic experience is not limited to one language. Logical possibilities include-but are not necessarily limited to - the following: (i) there is no transfer; (ii) the L1 is by default the privileged source of transfer; (iii) the L2 is by default the privileged source of transfer; (iv) either the L1 or the L2 may in principle be the source of transfer. While options (i) and (ii) have not been formalized as specific models in the literature (although see, e.g., Hermas, 2010, for some suggestions that the $\mathrm{L} 1$ is a default), a privileged status of the $\mathrm{L} 2$ and the equal availability of both languages have been articulated into three different models, two of which argue in favor of option (iv) albeit in different ways. Because these models are discussed in greater detail in the introduction to this special issue (Castro, González Alonso, Rothman and Westergaard, this issue), we will only briefly review them below.

\section{The L2 Status Factor}

The L2 Status Factor (L2SF; Bardel and Falk, 2007, 2012; Falk and Bardel, 2011; Falk et al., 2015) claims that the L2 is, by default, the privileged source of transfer throughout development in L3A. To the extent that transfer happens post the true L3 initial state, whether in whole, as seems to be the case in L2A (e.g. Schwartz and Sprouse, 1996) or in part, the L2 is argued to be the source. The main claim is based on the assumption that the L1 is less accessible than the 2 because of a fundamental difference in how non-native/non-primary languages are acquired in adulthood as compared to childhood. 


\section{The Cumulative Enhancement Model}

The Cumulative Enhancement Model (CEM; Berkes and Flynn, 2012; Flynn et al., 2004), which by the very nature of its claims is both an initial stages as well as developmental theory, does not envision any default grammar as contributing to the initial L3 interlanguage grammar and definitively rejects the notion of wholesale transfer. The idea is that the L3 interlanguage builds from the bottom up on a property-by-property basis and, as such, each time the L3 interlanguage grammar is ripe to add new properties each previous language, the L1 or L2, can contribute positively by transferring the relevant property. However, the CEM claims that this property-by-property influence will only happen when at least one of the previous grammars instantiates the property like the L3, thus transfer is not only developmentally incremental, but indeed only facilitative.

\section{The Typological Primacy Model}

The Typological Primacy Model (TPM; Rothman, 2010, 2011, 2013, 2015), like the CEM, does not restrict the source of transfer to any default, the L1 or the L2. The model is very clear on its position as to the completeness of transfer, that is, the TPM argues for complete, wholesale transfer of either the L1 or L2 as the initial L3 interlanguage grammar. As such, it might be viewed as the only model to be uniquely an initial stages model. The TPM argues that the linguistic parser determines - for several reasons of linguistic, cognitive and learning economies (see Rothman 2013, 2015 for more in depth argumentation of why this should be so) — which of the two systems should be transferred guided by a set of implicationally hierarchical linguistic 
cues coming from the L3 input to which novice learners find themselves exposed. The general idea is that the parser must engage with the L3 from its initial exposure and that the linguistic evidence encoded in the L3 input (words, sounds, morphology, syntactic structure, in that order) is unconsciously assessed against the grammars of the L1 and L2 to determine which grammar is closest holistically and thus should be the best source for transfer. Following the basic tenets of the Full Transfer hypothesis for L2A of Schwartz and Sprouse (1996), Rothman (2013, 2015) articulates why wholesale transfer is desirable, yet slightly delayed in L3A with respect to L2A for reasons related to the added experience that multilinguals bring to the task of subsequent language acquisition (see also the penultimate section of the article, below).

\section{Linguistic transfer vs. cross-linguistic influence}

Before proceeding, it is important to clarify here the difference — as we see it—between transfer and cross-linguistic influence (CLI). Transfer, as we intend the construct, refers to the level of grammatical mental representation for a given property, whereas CLI could be a simple bleeding over in performance and/or processing - influence in the truest sense of the wordfrom outside the interlanguage system. Let us illustrate this with two examples. Upon initial exposure to English, sufficiently so that an initial interlanguage for English emerges, the initial L2 interlanguage hypothesis/representation of a native speaker of Spanish might be that, like the L1, English realizes gender agreement in the nominal domain. That is, the initial interlanguage grammar for L2 English would have all the necessary structure for syntactic gender to be realized. The property can then be said to have been transferred, or copied, from the L1 
representation. As we will discuss later on, part of the acquisition process of this learner will be to reconfigure her grammar after rejecting this hypothesis through repeated parsing failures, given that she will be unable to match the gender property to any particular set of English morphemes.

Another difference between Spanish and English can be found in relative clause attachment preferences. In (a prosodically neutral instance of) the sentence The killer stabbed the brother of the policeman who was in the restaurant, Spanish native speakers will generally interpret the relative clause 'who was in the restaurant' as referring to the DP 'the brother' (high attachment preference), whereas English native speakers will normally interpret it as referring to the DP 'the policeman' (low attachment; see, e.g., Carreiras and Clifton, 1999). A different relative clause attachment preference can be, and is in fact generally acquired by the time learners reach a high level of proficiency. So much so, in fact, that L2 parsing preferences of this kind can influence L1 processing in immersed, highly proficient L2 speakers. Dussias (2004) has shown that, after continuous exposure to this kind of ambiguities being resolved locally in English (i.e., low attachment), Spanish native speakers end up applying this attachment preference when processing their L1. This is, to our mind, a very clear case of CLI that does not involve any level of representational change, but rather concerns processing influence being exerted by one language on another (L2 > L1, in this case). Another, less systematic, example of CLI would be when a previously existing language exercises a temporal influence at any given moment that is not found pervasively in the holistic performance of a given speaker. Often referred to as slips of the tongue, such influence that can be likened to another known language might underdetermine the actual target representation of the L2/L3 speaker. These slips, however, 
should not be surprising since the speaker, after all, has at least one more grammar that must be suppressed while actively engaging in the other/target grammar. Determining what is true transfer (i.e., representational) and what is CLI is, in principle, empirically feasible as they map onto the constructs of competence vs. performance variables nicely. Although both stem from previously acquired grammars, transfer maps onto linguistic competence whereas CLI maps onto performance.

The distinction between transfer and CLI is a very relevant one to be made, because (i) as formal linguists we are primarily interested in describing and explaining mental representations, and (ii) being able to make predictions for L3 learnability inherently refers to the path of transitions in mental representations within L3 interlanguage grammars over time. It is prudent to point out a fact that pertains to all models, at least as we understand them: none precludes true CLI (and even transfer) from the previous languages at later stages of development. In the case that a model argues that initial transfer is wholesale from one system, as the TPM does, it does not necessarily follow that it envisions a blocking of subsequent CLI and transfer at later stages. What happens beyond the initial representations of L3 interlanguage grammar is outside the remit of models like the TPM because it is a question of L3A itself. L3 initial stages models are not really about L3A per se, but rather about providing an accurate portrayal of the point of departure for L3A. In other words, L3 initial stages models are best understood as hypotheses regarding the initial L3 interlanguage representation.

\section{The importance of the L3 initial stages and moving forward}

Modeling accurately the initial stages of L3A is the first step towards constructing a framework that will yield an empirically supported and theoretically informed comprehensive theory of L3 
development. Why? Because just like a building rests on the strength of its foundation, so do developmental theories depend on the strength of understanding the starting point of development. Given that the field of L3 morphosyntax is still in its formative years, it seems sensible to continue the process of testing and vetting various hypotheses about the initial stages before attempting to offer a comprehensive theory. Such a statement is not meant to discourage work in L3 morphosyntax past the initial stages. To the contrary, a further necessary building block for any eventually viable complete theory of L3A is a strong base of empirical evidence from L3 development itself. Our point here is twofold. First, it is not appropriate in most cases - a point to which we return in detail below - to use developmental data to adjudicate between competing initial stages theories. Second, since it would be uncontroversial to claim that $\mathrm{L} 3 \mathrm{~A}$ as a process is complex and dynamic, it is best to understand the starting point as completely as possible before modeling its outcome. In the absence of a complete theory of L3A, however, this does not mean that the field is left without knowing where to begin a focused effort to collect developmental data needed to transition towards comprehensive theories. Fortunately, there are (limited) developmental predictions that stem directly from the initial stages models. We contend that testing these developmental predictions is the most immediately fruitful path to expand the scope of L3 morphosyntactic research. Doing so offers benefits on at least two fronts: (i) it provides a new means to tease out the best model of the initial stages, and (ii) it offers at the same time developmental insights that easily relate to the focus of the field as it is. Before developing this line of argumentation in greater detail below, we first discuss some theoretical issues related to the construct of initial stages in L3A.

\section{On the construct of initial stages}


To date, formal linguistic models have been either uniquely or mostly focused on determining the source of linguistic transfer in the earliest stages of L3A. As we have claimed, this is an obvious and reasonable approach because successfully explaining the trajectories of L3A is inherently related to understanding accurately how previous linguistic experience factors into the earliest L3 stages. Defining, however, what constitutes the initial stages of L3A in actual time - by hours, days or weeks - is by no means an easy task and, we submit, likely not a fruitful endeavor to fixate on because many factors likely interact to determine its onset and length depending on context, access to input (quality and quantity), the structural relationship of the languages in the triad and what type of bilingual (e.g., early vs. late) the L3er is, among others (see Rothman, 2013, 2015 for discussion). Ultimately, stating in absolute terms that the initial stages is wholesale $\mathrm{X}$ amount of time after initial exposure would only serve to satisfy an immediate need or desire to have a definition. At the same time, providing a catchall figure would effectively deny the dynamic linguistic and cognitive nature of multilingualism itself (see Green, this issue). At first glance, it might seem problematic, if not overly convenient for certain theories, to not have a definitive timeframe for the L3 initial stages. However, if the reality is that both the onset and/or length of the initial stages depend on multiple factors such that it is not exactly the same in all scenarios, then providing an arbitrary number would be counterproductive. Instead of offering an absolute timeframe for L3 initial stages, we would rather shift the locus of its definition away from calculable/absolute time towards a linguistically defined one. The goal is to tap the mental representation of the initial L3 interlanguage grammar and possibly the first few instantiations of subsequent interlanguage grammars along L3 development. Therefore, we believe that researchers should instead 
document the amount of input and time of exposure that learners have received, and argue why the observed stage reflects the initial interlanguage grammar in consideration of the applicable variables for any given reported group.

Unlike L2A, where the same issues of temporality related to the initial state and defining the earliest L2 interlanguage development have existed for decades, L3A is more nebulous because of the multiple sources of previous linguistic systems and the plethora of cognitive and linguistic factors that interact to determine how transitional development will take place from a starting point of “more" as compared to L2A (see Rothman, 2013, 2015). Potentially, individual differences outside the strict domain of linguistic variables (e.g., in working memory and/or other cognitive aspects) play an even greater role in determining transitional timing for L3A, for example, related to the experience of bilingualism individual L3 learners have had (see Green, this issue).

From the perspective of the above models, the elusiveness of defining in absolute terms the initial stages is not necessarily problematic. In the L2SF, transfer is predicted to begin taking place upon first exposure to the L3, and so it should be detectable from early on. For the TPM, the initial stages are themselves defined as the period in which structurally driven wholesale transfer from the L1 or the L2 takes place. In the case of the CEM, instances of transfer will happen more often at earlier stages in L3 interlanguage development, and will likely decrease as an inverse function of proficiency. In any case, an implied assumption of all models is that, in principle, the further ahead in (L3) interlanguage development we test our participants, the harder it will be to determine backwards whether the observed performance is reflecting a transferred property from the L1/L2 or a property already in transition towards or at the L3 
target as a result of L3A itself. A methodological precaution follows straightforwardly: if the goal is to determine the transfer source in any given L3 study, then the aim should be to test participants as close as possible to first exposure.

\section{Developmental stages data: drawbacks and advantages}

Moving forward, we highlight the potential pitfalls of using later stage data backwards to comment on the validity of initial stages models, inclusive of when it could be appropriate-i.e., when one probes specifically for the developmental predictions of the models themselves. Some studies have used data from intermediary stages of L3A to comment on the three available models. Like all datasets obtained through experimentally sound procedures, these are illuminating and worthy of serious consideration — ultimately, we seek to understand all data. However, it is not always the case that all datasets are appropriate for particular inquiries. The main issue with using developmental stages data for assessing initial stages modelsspecifically with reference to the TPM-is the lack of temporal control one has over the variables that determine the nature of the dataset-i.e., the complex variables that pertain to acquisition itself. Recall, for example, that the aim of the TPM is to describe the original L3 interlanguage grammar-i.e., the point of departure for acquisition, not L3A itself. Inherently, any claims made about the beginning of a process from data well past the point of onset entail backwards reconstruction of the process. It presupposes that other dynamic variables, which no one denies likely apply to L3A, can be ignored or meaningfully teased out, or did not apply in the first place. However, there is of course no way to definitively know.

To be sure, developmental stages data present a different, perhaps more complex sourcing problem from initial stages data: it is difficult, if not impossible, to determine which instances of 
interlanguage carry over from transfer at the initial stages, and which reflect the process of learning/acquisition that has taken place since. It would be overly convenient to simply dismiss all data not obtained at the initial stages, especially when in seeming disaccord to a particular model. To be clear, we are not advocating such a position. It is our belief, however, that only methodologies specifically designed to obtain appropriate developmental stages data should be used towards characterizing the initial stages of L3A. When the dynamic variables pertaining to the L3A process itself cannot be meaningfully teased out, such datasets should not be used to comment on the initial stages models.

When can data from intermediary L3 proficiency levels still be taken as a valid testing ground for predictions of initial stages models? The most uncontroversial answer to this question should be when such data form part of a longitudinal study. The best way to tap the initial interlanguage grammar(s) is to test and track ab initio learners. To date, exceedingly few studies have done this, although many have examined learners quite early on (a few days or weeks after first exposure). To our knowledge, there are no studies that start data collection at the initial stages and track these same L3 learners over developmentally long periods of time. Longitudinal studies that exist either use a cross-sectional design (e.g., Cabrelli Amaro, 2015; Cabrelli Amaro et al., 2015) or chart the same learners over time starting definitively after the initial stages - in some cases, after one or more years of exposure to the L3 (e.g., García Mayo and Villarreal Olaizola, 2011; Sánchez, 2015). Examining L3A in a laboratory using microgrammars or artificial language might be a good way forward in this regard (see, e.g., Rothman et al., 2015; Sanz et al., 2015). However, generalization from artificial grammar learning scenarios is meant, at best, to complement more ecologically valid designs of (pseudo) real 
world acquisition. Not only would true longitudinal studies provide the most definitive means to confidently relate developmental stages data to initial stages models, but indeed they are the ideal scenario to start to form theories of L3A. Why? Because longitudinal studies would not have to make any assumptions regarding what previous L3 interlanguage grammars looked like. Rather, their very design would entail the charting of development from the outset of L3A. Nevertheless, we acknowledge that doing longitudinal studies is not universally practical.

There is a second logical scenario under which developmental data can be used to relate back to initial stages models: following the learnability predictions stemming from the hypothesized source of transfer that each model makes. Indeed, in predicting the source of L3 transfer, the initial stages models crucially also make clear developmental predictions, since they effectively hypothesize the initial L3 interlanguage representations for any given property. From the initial point of transfer, however, all initial stages models - to our understandingagree that acquisition can and will take place. From that initial transfer point, the same linguistic constraints $^{1}$ that pertain to learnability in all instances of language acquisition (e.g., L1A, L2, 2L1A) should equally apply to L3A (Rothman, 2013, 2015). Perhaps, then, the main innovative feature that L3 initial stages models underscore is that not all native speakers of any given language will have the same path for non-native language acquisition. More concretely, an L1 speaker of English acquiring Spanish as an L2 may have a significantly different path to learning Spanish than an L1 English speaker of L3 Spanish who already speaks Italian. An L1 English speaker of L3 Spanish who knows French or a child heritage speaker of Spanish, dominant in English learning the formal register of Spanish as an L3 (see Polinsky, 2015) might each be different from the other learner types despite the fact English is the first/dominant 
language and Spanish is always the target. Among other variables that will likely intercede along the path of linguistic development in L2A/L3A, what makes the learnability task potentially different for each of these native speakers of English always acquiring standard Spanish as a non-native language is how the initial transferred representations, predicted to be (somewhat) unique in each case, translate into differences in learnability for interlanguage development. Whether you have French or Italian as an L2 could make all the difference in the L3 acquisition of Spanish, not only at the beginning but indeed developmentally, as predicted by the TPM and discussed in further detail below.

Developmental predictions stemming from models like the TPM, we believe, are exactly the types of properties we should first focus on in studies examining later stages of L3A, for two main reasons. The first is that these predictions relate to the initial stages models, and thus can be properly used to falsify them. The second is that they derive from an interaction of the predicted initial stages representations and linguistic constraints on learnability. To illustrate the main points we have made thus far, we bring together two studies looking at properties related to the licensing of null-subjects in different language triads, and highlight relevant insights.

In Lacroix (2015), null subject-related properties were examined in the production of intermediate L3 Spanish learners. For the purpose of the present discussion, the mirror image groups of L1 Basque-L2 French-L3 Spanish and L1 French-L2 Basque-L3 Spanish are of significance. Basque, the typologically least similar language of the two to Spanish, does in fact license true null subjects like Spanish (i.e., pro), whereas French, the clear typologically similar language, does not. The TPM predicts, for both groups, that French is transferred at the initial stages irrespective of its order of acquisition. The expectation, therefore, is that null subjects 
would not be licensed as part of the initial L3 Spanish interlanguage grammar. If the L2 were transferred by default, as the L2SF proposes, the L1 French-L2 Basque learners should be the only group that has an initial L3 interlanguage grammar that licenses null subjects. The CEM would predict that both groups, since they speak Basque, would have an initial L3 Spanish interlanguage grammar that licenses null subjects. The experimental task was Frog, where are you?, a picture-book storytelling production task. The pattern of results in Lacroix (2015) shows that both groups approximate targetlike Spanish distribution of null subjects, with very few instances of redundant overt subjects. At first glance, given the performance of the groups which approximate in both cases convergence on the Spanish target, one could draw two conclusions. Either Basque was transferred irrespective of order of acquisition, or both groups transferred French initially and have already reconfigured their Spanish grammar towards the target representation. The fact that these are intermediate learners, as discussed below, means that we cannot adjudicate between these two possibilities.

Rothman and Cabrelli Amaro (2010) examined null subject related properties in L2 and L3 acquisition of French and Italian as target languages. In all four groups, the native language was English and either Italian or French was the target L2/L3. Crucially, each individual was at the very beginning of L2/L3 acquisition. What differentiated the L2/L3 French and Italian groups was that in the case of L3 French and L3 Italian, Spanish was a successfully acquired L2. They examined various properties related to null subject pronouns. Focusing on the L3 groups, for the domain of null subject licensing, the TPM predicts that both groups will transfer Spanish and thus have an initial L3 interlanguage grammar that licenses null subjects, even if this does not correspond to the target property in L3 French. The L2SF also expects Spanish-the L2 in 
both groups - to be transferred, with the same consequences. The CEM predicts transfer from whatever language matches the target L3 property. In the case of L3 French learners, this would result in transfer from English yielding an initial L3 interlanguage grammar with no null-subject licensing. In the case of L3 Italian, this would result in transfer from Spanish yielding an initial L3 interlanguage grammar that licenses null subjects. The data revealed that the L2 groups performed identically, showing influence of English (the only available option for transfer). The L3 learners could not be distinguished from each other either, yet were completely different from the L2 groups, showing transfer from Spanish. While the design cannot adjudicate between the TPM and the L2SF- because typology and L2 status are confounded-, the results clearly present counterevidence to the CEM's predictions. To ameliorate the confound, mirror image L3 groups of L1 Spanish-L2 English-L3 French/Italian would be required.

These two studies, illuminating as they are, present one difference that assigns crucial importance to the stage at which observations are made. Taken at true initial stages, data from both designs - ideally, if both had mirror-image groups - would be maximally informative to adjudicate between the potential constraining factors advocated by the CEM, the TPM and the L2SF. If participants have moved beyond these first steps, however, targetlike performance might reflect transfer as much as true acquisition of the property. Going back to Lacroix (2015), if at the intermediate level of L3 Spanish, L1 French-L2 Basque learners are correctly producing null subjects in certain contexts, can one unequivocally claim that they have transferred L2 Basque? If both this group and their mirror image (L1 Basque-L2 French) seem to have targetlike knowledge of null subject-related properties, should one assume that French was never transferred, and that Basque was the selected source for reasons of maximal facilitation? 
The answer, to both questions, is no. As we know from the L2A literature, the acquisition of the syntax of null subjects by native speakers of non-pro-drop languages (e.g., L1 English learners of L2 Spanish) happens relatively early and is largely successful (e.g., Liceras, 1989; Rothman, 2009). That is, transfer of a non-null subject syntax does not block target acquisition in nonnative language acquisition, since there is no learnability problem. Parsing failures follow naturally, because a transferred syntax which does not license null subjects cannot parse sentences that do not overtly realize subjects. As a result, the initial transferred representation must be revised in subsequent stages of interlanguage development to accommodate the target input. It follows from such evidence that, even if the L1 Basque-L2 French and the L1 FrenchL2 Basque learners had transferred French in the initial stages of L3 Spanish, it is possible for them to acquire this property through the course of L3A, and thus have the same knowledge of subjects by the time they reach intermediate proficiency that they could have gotten from transferring Basque. In other words, it would not be appropriate to comment on the above models based on the performance of Lacroix's (2015) learners, precisely because past the initial stages Basque transfer cannot be reliably distinguished from true acquisition of L3 Spanish.

Crucially, this same argument is not true of the second design (Spanish-English learners of L3 French) for reasons well known to formal linguistic theory. The grammars of languages presenting null and obligatory overt referential subjects, respectively, stand in a subset/superset relationship: all sentences with overt referential subjects are grammatical in a null subject (prodrop) language, but no sentences with syntactically licensed null subjects (with a pro subject) are acceptable in an overt subject (non-pro-drop) language - the grammar of the pro-drop language thus being the superset. Evidence from L2A suggests that it is very difficult, if not 
impossible, for learners to reconfigure an initial grammatical representation that is a superset to the target L2 representation. For example, Phinney (1987) and Judy and Rothman (2010) have both shown that even advanced speakers of L2 English whose L1 is Spanish, show lingering evidence of L1 transfer of null subject phenomena. Such evidence runs in sharp contrast to the relative success in acquiring the syntax of null subjects in L2 Spanish with English as an L1 as referenced above. The difference in directionality, however, is not surprising when learnability constraints are considered stemming from what the initial interlanguage representation is hypothesized to be. Assuming L1 initial transfer in both cases, Spanish $\rightarrow$ English is predicted to be harder than English $\rightarrow$ Spanish. So, even in the case one is a native speaker of English, if as a successful L2 learner of Spanish she transfers Spanish instead of English as an initial L3 representation then this learner should follow the developmental predictions of a Spanish native, not an English one. In terms of the previously discussed studies, if Rothman and Cabrelli Amaro's (2010) English-Spanish learners of L3 French transfer Spanish-for reasons of L2 status, typological proximity or otherwise - they will have a much harder time recovering from the error this entails in the subject domain than Lacroix's (2015) Basque-French learners potentially transferring non-pro-drop syntax into Spanish. The reason is that positive evidence of overt subjects would not force this second group of learners to abandon their hypothesis that French is, like Spanish, a null-subject language, since these sentences would be perfectly acceptable in their interlanguage grammar. As a consequence it is likely that, even if observed at intermediate stages, targetlike performance in the (overt) verbal subjects of Spanish-English learners of L3 French indicates transfer from English rather than true acquisition of the property. 
The reason is that, if non-facilitative transfer from Spanish obtains at the initial stages, the subset/superset problem will make it harder to overcome until well into more advanced stages.

We hope that the above discussion persuades the reader of three things. The first is that L3 initial stages transfer models (e.g., TPM) are best tested with data from true initial stages or, alternatively, with intermediate stages data that leave their predictions unconfounded with the effects of acquisition. The second is that this is also true of models that do not necessarily focus on the initial stages (e.g., L2SF, CEM), because their predictions for transfer will increasingly be confounded with true acquisition. Finally, the third is that, from the initial stages, all these models do contain implicit-if formally unarticulated-predictions for further developmental stages. These can be inferred and tested by following through the initial predictions, and drawing from insights of formal linguistic theory to detect where and how transfer effects are expected to interact with true language acquisition.

\section{Conservatism in building a complete theory of L3 morphosyntax}

The fact that initial stages models of L3 morphosyntactic transfer endeavor to make overt predictions only for the selective nature of transfer in the first instance-and are thus best tested at the initial stages — and/or make strong claims about the completeness of it, has been recently highlighted as a limitation (Slabakova, this issue; Westergaard et al., this issue). In summary, it is claimed that focusing on the initial stages and/or taking the position that transfer is initially complete in nature from a single source fails to consider the very dynamic nature of possibly deterministic variables in L3A. While true in the most abstract sense, critiquing models for not directly engaging something that is outside their intended remit or for taking a strong position 
that is seemingly justified by available data sets is perhaps injudicious. Related to the argument of completeness in transfer, it is useful to point out that the TPM's position follows directly from L2 acquisition theory, where hundreds of studies over the past three decades seemingly point to this possibility, as well as considerations of linguistic, cognitive and learning economies as discussed in Rothman (2013, 2015). However, and more importantly, it is also justified by the strength of independent evidence of complete transfer in various L3 studies when considered as a whole. Although one must acknowledge that the language pairings in existent L3 data sets are presently limited and comparatively scarce in relation to other scenarios of acquisition, those that can be brought together seem to point in a single direction. Within the limited cohort of available L3 data sets, the largest number involve L1/L2 Romance-L1/L2 Germanic/NonRomance-L3 Romance. In these pairings, an impressive range of structures have been examined, which all seem to point to the previous Romance language as the transfer source to the Romance L3 target, whether an L1 or L2 and whether or not such transfer was facilitative. Properties examined include, but are not limited to: raising structures (Cabrelli Amaro, Amaro and Rothman, 2015), dislocation structures (Slabakova and García Mayo, 2015), grammatical aspect (Foote, 2009), differential object marking (Giancaspro, Halloran and Iverson, 2015), articles (Ionin, Montrul and Santos, 2011), the syntax of DPs (Iverson 2009, 2010; Rothman 2011), clitic pronouns (Llama, 2013; Montrul, Dias and Santos, 2011), existential constructions (Núñez, 2011), referential subjects (Rothman and Cabrelli Amaro, 2010) and word order (Rothman, 2010), etc. In light of this, it seems that complete transfer is well justified as an initial hypothesis to be rejected upon an accumulation of counter evidence to what these studies seem to suggest individually and, crucially, when considered together. 
Slabakova (this issue) further points out that one wants to understand all the variables involved, linguistic and otherwise, in L3A. For example, she highlights that additional factors such as processing complexity, misleading input, negative evidence and construction frequency in the target L3 might play an important role in explaining time course, development and ultimate attainment (see Slabakova, this issue, for details). Even if we are centrally focused on trying to understand the nature of the initial L3 interlanguage grammar, one really wants to know whether, in Slabakova's terms, the cognitive and psychological prominence (native, adultonset or child-onset, strong additional or weak additional language, etc.), the typological characteristics or the linguistic characteristics of the languages acquired play a decisive role in CLI/transfer (see also Rothman, 2015, and Green, this issue, for a discussion of some of these variables).

To be sure, we could not agree with Slabakova more. Her contention that all of these variables are probably co-existing and conspiratorial is most likely on the right track. Of course, the extent to which is an open empirical question which currently lacks the necessary data to assess. The field also wants to know when and why in the course of L3A (probably at multiple instances) CLI/transfer obtains, what shape it takes in the entirety of the process and what its consequences are as seen in developmental data. Recall, as discussed above, that the TPM has never stipulated that CLI and/or transfer cannot happen at subsequent stages of L3 development; it simply has never commented on it since by definition such would obtain outside of its remit, that is, outside the initial stages. In fact, we are inclined to argue and put on record that the null hypothesis would be that transfer does happen at later developmental stages. If subsequent influence can come from other previously acquired languages upon further evidence from the 
target L3, this would make L3A more unique from L2A than currently demonstrable. For L2A, to the extent that L1 transfer happens, the only recovery from it, when non-facilitative, would be acquisition of the target. For L3A, the possibility that recovery from non-facilitative transfer could entail accessing the other language not initially transferred might predict, when appropriate given the linguistic property, the languages involved and context, that L3A will be predictably faster for some properties than L2A. Of course, this same scenario can work in reverse, and formal linguistic theory can help us make predictions of when. L3A is arguably a more complex process than L2A, and one that is, for the moment, much less documented -an artifact we must accept given the youth of the field. Our main point is that, before we attempt to offer a holistic L3 theory from piecemeal and insufficient amounts of data, pursuing smaller yet theoretically informed hypotheses that make falsifiable predictions might be more fruitful in precisely fostering the collection of sufficient amounts and types of the specific data needed.

As it turns out then, the issue is not whether we want to understand the full dynamic nature of L3A, but rather whether we are prepared to do so at this point. Scientific prudence requires that new theories not only push the envelope of current thinking and draw attention to holes in the path towards a desired goal—even when that part is excellently argued — but they too must also provide a manner in which their contentions can be tested. In other words, a theory, to be a theory, must be falsifiable empirically. Slabakova's Scalpel Model, although being the first to point out many interesting variables that ultimately will form the basis of a complete theory of L3A, does not seem to meet the minimum definition of a theory proper. True as her observations likely are, it is not immediately obvious how one takes the general approach and derives predictions experimentally. 
Other models, such as the CEM, TPM and L2SF do make clear predictions, falsifiable in their own right and especially against one another. The question 'What do the TPM, L2SF and the CEM predict for the transfer source of property $\mathrm{X}$ in language triads $\mathrm{ABC}$ and $\mathrm{BAC}$ ?' is easily answered. Being mutually exclusive in their predictions, only one can be right. Given the infancy of the field, it is likely they are all wrong. Crucially, however, they each meet the minimum requirement of a theory: prediction and falsifiability. Our goal is not to critique the Scalpel Model per se. As we have already conceded, many of its tenets are welcome additions to the field because they highlight what the eventual end goal is for the study of L3A and the complex nature of the road ahead. From our perspective, no comprehensive theory, at this point in time, could do a better job because of the infancy of the field and the dearth of data we have, especially compared to other fields like L2A, which also lacks a comprehensive complete theory despite many more decades of practice and available data.

Our primary purpose in discussing the Scalpel Model, and indeed the general point that we are trying to make in this paper, are perhaps best understood by considering the basic nature of scientific modeling. In a standard modeling process, the individual and combined effects of different variables on an observed measure are estimated, so that the model can predict a value for the observed measure when the influencing variables take on values that have not been attested. Generally speaking, models benefit from as many observations as possible, since these permit gradual adjustment of the estimated effects and, on the whole, yield a more accurate model that can make more precise predictions. The main weakness of the Scalpel Model is that, while sharply identifying potential variables that may have an effect on the outcome and 
development of the L3A process, it does not attempt to provide an estimate of those effects, and so it is unable to offer reliable predictions.

A shared concern of proposals such as the Scalpel Model or the Linguistic Proximity Model is that the idea of initial stages wholesale transfer proposed by the TPM underestimates the subtlety of the language acquisition process, and suggests that learners are only sensitive to macro-cues (e.g., subject drop), as opposed to micro-cues (Westergaard, 2009). However, as stated above, there is no indication that advocating initial wholesale transfer means that models such as the TPM reject the idea of transfer beyond the initial stages. This process is hypothesized, first, on the basis of a considerable amount of empirical evidence (see above) and, second, for a number of cognitive reasons that have been discussed in previous work on these models (see Bardel and Falk, 2012; Rothman, 2013, 2015), and its outcome is assumed to be the initial L3 interlanguage grammar. Since wholesale transfer is taken to happen at a very early stage, the greater saliency of macro-cues is expected to better serve the purpose of making an informed estimation as to what the source should be. Of course, micro-cues are most probably in operation along L3 interlanguage development. However, we believe that their effect is best estimated on the basis of a still picture of L3 interlanguage that wholesale transfer theories provide hypotheses for, at least in initial stages learners.

It is important to clarify, however, that our methodological point stands and remains relevant for property-based and wholesale transfer models alike. Whether or not one assumes that transfer takes place in whole at some early stage after initial exposure, it is undeniable that more instances of (unassisted) true acquisition will take place with the progression of L3 interlanguage development. This fact, which does not preclude instances of transfer or CLI at 
later stages, makes it progressively harder to unambiguously determine whether a given property has been transferred from previous languages or "simply" acquired anew. For this reason, and irrespective of the variables we believe to condition syntactic transfer, data from initial stages learners has the greatest chance to offer unconfounded insights into the mechanics of this process.

\section{Conclusions}

This article has focused on two aspects related to the burgeoning field of L3 morphosyntax. The first concerns the suitability of datasets used for the empirical assessment of L3 initial stages models. We have highlighted how target acquisition at later stages cannot be easily distinguished from confounds related to transfer source. As a result, we have argued that not all data from intermediary stages of L3A provides unequivocal insight into the original L3 interlanguage grammar, but crucially some data can. We contend that following the implied developmental predictions of initial stages models not only constitutes the type of later stage data that can be used to adjudicate between competing initial stages models, but is also, in our view, the most productive way of starting the process of charting and characterizing developmental L3 interlanguage grammars. These predictions, we believe, can be derived by combining those from initial stages models with insights from formal linguistic theory.

The second, more general aspect follows naturally from the first. Because we believe that the best road to building an eventual complete theory of L3A is by modeling from the bottom up, given the complexities of L3A and our general novice state of knowledge, we have closed our discussion with a call for conservatism in the field's attempts to work towards comprehensive 
theories of L3A. Our goal is not to stop new theories from emerging, nor blocking emerging ones from being tested. Our goal is simply to point out where we believe the best path is to be found, which we take to be incremental growth stemming from testable hypotheses that minimize confounds resulting in a lack of testability/falsifiability. To the extent that our call for conservatism is well-received, we should highlight various immediate domains that the field should also focus on to fill the gaps needed to progressively move forward. Indeed, many of these aspects ${ }^{2}$ have been or are currently being considered in several studies, and include:

(i) data from less-studied language combinations,

(ii) greater control of participants' L1/L2 knowledge of the domain tested in the L3,

(iii) data from larger groups of L3 learners,

(iv) data from a larger range of morphosyntactic properties, and

(v) data from both comprehension and production tasks, online and offline.

These criteria should help guarantee that we obtain the much needed data from initial stages studies meeting minimum standards of generalisability, replicability and falsifiability.

\section{Notes}

1. We specifically refer here to linguistic constraints, for example learning principles such as the Subset/Superset Principle (e.g., Berwick, 1985; Borer and Wexler, 1987). Such a statement does not imply that other variables affecting acquisition are the same for all types of learners or that particular learning principles themselves are uncontroversial. It is intended as an example. Clearly, other variables such as metalinguistic knowledge, cognitive factors, etc. will be different for distinct types of learners and indeed across individuals of a given 
type (see Green, this issue). Moreover, some assumed learning principles are likely wrong. Nevertheless, the larger point we endeavor to make stands irrespectively.

2. The list we provide here has been suggested by an anonymous reviewer, whom we thank for summarizing some of the most important methodological criteria L3 initial stages studies should, in our opinion, ideally follow. 


\section{References}

Bardel C and Falk Y (2007) The role of the second language in third language acquisition: the case of Germanic syntax. Second Language Research, 23(4), 459-484.

Bardel C and Falk Y (2012) Behind the L2 Status Factor: A neurolinguistic framework for L3 research. In: Cabrelli Amaro J, Flynn S, and Rothman J (eds), Third Language Acquisition in Adulthood, Amsterdam: John Benjamins, pp. 61-78.

Berkes É and Flynn S (2012) Further evidence in support of the Cumulative-Enhancement Model. In: Cabrelli Amaro J, Flynn S, and Rothman J (eds), Third Language Acquisition in Adulthood, Amsterdam: John Benjamins, pp. 143-164.

Berwick R (1985) The acquisition of syntactic knowledge. Cambridge, MA: MIT Press.

Borer H and Wexler K (1987) The maturation of syntax. In: Roeper T and Williams E (eds), Parameter setting, Dordrecht: Reidel, pp. 123-172.

Cabrelli Amaro J (2015) Does the source of transfer affect the rate of L3 morphosyntactic development? Paper presented at the 40th Boston University Conference in Language Development, Boston, MA.

Cabrelli Amaro J, Amaro F and Rothman J (2015) The relationship between L3 transfer and structural similarity across development. In: Peukert $\mathrm{H}$ (ed.), Transfer effects in multilingual language development, Amsterdam: John Benjamins, pp. 2-23.

Carreiras M and Clifton C (1999) Another word on parsing relative clauses: eyetracking evidence from Spanish and English. Memory \& cognition, 27(5), 826-833.

Castro T, González Alonso J, Rothman J and Westergaard M (this issue) Introduction (Title TBA). International Journal of Bilingualism.

Dussias PE (2004) Parsing a first language like a second: The erosion of L1 parsing strategies in Spanish-English Bilinguals. International Journal of Bilingualism, 8(3), 355-371.

Falk Y and Bardel C (2011) Object pronouns in German L3 syntax: Evidence for the L2 status factor. Second Language Research, 27(1), 59-82.

Falk Y, Lindqvist C and Bardel C (2015) The role of L1 explicit metalinguistic knowledge in L3 oral production at the initial state. Bilingualism: Language and Cognition, 18(02), 227235. 
Flynn S, Foley C and Vinnitskaya I (2004) The cumulative-enhancement model for language acquisition: Comparing adults' and children's patterns of development in first, second and third language acquisition. International Journal of Multilingualism, 1(1), 3-16.

Foote R (2009) Transfer and L3 acquisition: the role of typology. In: Leung YI (ed.), Third Language Acquisition and Universal Grammar, Clevedon: Multilingual Matters, pp. 89114.

García Mayo MP and Villarreal Olaizola I (2011) The development of suppletive and affixal tense and agreement morphemes in the L3 English of Basque-Spanish bilinguals. Second Language Research, 27, 129-149.

Giancaspro D, Halloran B and Iverson M (2015) Transfer at the initial stages of L3 Brazilian Portuguese: A look at three groups of English/Spanish bilinguals. Bilingualism: Language and Cognition, 18(2), 191-207.

Green D (this issue). Trajectories to L3 proficiency. International Journal of Bilingualism.

Hermas A (2010) Language acquisition as computational resetting: verb movement in L3 initial state. International Journal of Multilingualism, 7(4), 343-362.

Ionin T, Montrul S and Santos H (2011) Transfer in L2 and L3 acquisition of generic interpretation. In: Danis N, Mesh K, and Sung H (eds), BUCLD 35: Proceedings of the 35th annual Boston University Conference on Language Development, Sommerville, MA: Cascadilla Press, pp. 283-295.

Iverson M (2009) N-drop at the initial state of L3 Portuguese: Comparing simultaneous and additive bilinguals of English/Spanish. In: Pires A and Rothman J (eds), Minimalist inquiries into child and adult language acquisition: Case studies across Portuguese, Berlin: Mouton de Gruyter, pp. 221-244.

Iverson M (2010) Informing the age of acquisition debate: L3 as a litmus test. International Review of Applied Linguistics in Language Teaching (IRAL), 48, 221-243.

Judy T and Rothman J (2010) From a Superset to a Subset Grammar and the Semantic Compensation Hypothesis: Subject Pronoun and Anaphora Resolution Evidence in L2 English. In: Franich K, Iserman KM, and Keil LL (eds), 34th Boston University Conference on Language Development, Sommerville, MA: Cascadilla Press, pp. 197-208.

Lacroix H (2015) Syntactic transfer from French and Basque to L3 Spanish: The Null-Subject Parameter. Paper presented at the Workshop on Multilingual Acquisition, Processing and Use, Troms $\varnothing$, Norway. 
Liceras JM (1989) On some properties of the 'pro-drop' parameter: Looking for missing subjects in non-native Spanish. In: Gass S and Schachter J (eds), Linguistic Perspectives on Second Language Acquisition, Cambridge: Cambridge University Press, pp. 109-133.

Llama R (2013) Object clitic (mis)placement in L3 Spanish: Exploring the differences between the English-French and French-English Bilinguals. Paper presented at the II Coloquio de estudiantes de estudios hispánicos, Montreal, Canada.

Montrul S, Dias R and Santos H (2011) Clitics and object expression in the L3 acquisition of Brazilian Portuguese: Structural similarity matters for transfer. Second Language Research, 27, 21-58.

Mykhaylyk R, Mitrofanova N, Rodina Y and Westergaard M (2015) The Linguistic Proximity Model: The case of Verb-Second revisited. In Grillo E and Jepson K (eds): BUCLD 39: Proceedings of the 39th Boston University Conference on Language Development, Sommerville, MA: Cascadilla Press, pp. 337-349.

Núñez C (2011) The acquisition of existentials in L3 Spanish by speakers of Romanian and Serbo-Croatian. Paper presented at the Hispanic Linguistics Symposium, Athens, Georgia (USA).

Phinney M (1987) The Pro-Drop Parameter in Second Language Acquisition. In: Roeper T and Williams E (eds), Parameter setting, Reidel, pp. 221-238.

Polinsky M (2015) When L1 becomes an L3: Do heritage speakers make better L3 learners? Bilingualism: Language and Cognition, 18(2), 163-178.

Rothman J (2009) Pragmatic deficits with syntactic consequences? L2 pronominal subjects and the syntax-pragmatics interface. Journal of Pragmatics, 41(5), 951-973.

Rothman J (2010) On the typological economy of syntactic transfer: Word order and relative clause high/low attachment preference in L3 Brazilian Portuguese. International Review of Applied Linguistics in Language Teaching (IRAL), 48(2-3), 245-273.

Rothman J (2011) L3 syntactic transfer selectivity and typological determinacy: The Typological Primacy Model. Second Language Research, 27, 107-127.

Rothman J (2013) Cognitive economy, non-redundancy and typological primacy in L3 acquisition: Evidence from initial stages of L3 Romance. In: Baauw S, Dirjkoningen F, and Pinto M (eds), Romance Languages and Linguistic Theory 2011, Amsterdam: John Benjamins, pp. 217-247. 
Rothman J (2015) Linguistic and cognitive motivations for the Typological Primacy Model (TPM) of third language (L3) transfer: Timing of acquisition and proficiency considered. Bilingualism: Language and Cognition, 18(2), 179-190.

Rothman J and Cabrelli Amaro J (2010) What Variables Condition Syntactic Transfer? A Look at the L3 Initial State. Second Language Research, 26, 189-218.

Rothman J, Alemán Bañón J and González Alonso J (2015) Neurolinguistic measures of typological effects in multilingual transfer: Introducing an ERP methodology. Frontiers in Psychology, 6(1087), 1-14.

Sánchez L (2015) L2 activation and blending in third language acquisition: Evidence of crosslinguistic influence from the L2 in a longitudinal study on the acquisition of L3 English. Bilingualism: Language and Cognition, 18(2), 252-269.

Sanz C, Park H in and Lado B (2015) A functional approach to cross-linguistic influence in ab initio L3 acquisition. Bilingualism: Language and Cognition, 18(2), 236-251.

Schwartz BD and Sprouse RA (1996) L2 cognitive states and the Full Transfer/Full Access model. Second Language Research, 12, 40-72.

Slabakova R (2015) The Scalpel Model of Third Language Acquisition. Paper presented at the Workshop on Multilingual Acquisition, Processing and Use, Troms $\varnothing$, Norway.

Slabakova R (this issue) The Scalpel Model of Third Language Acquisition. International Journal of Bilingualism.

Slabakova R and García Mayo, MP (2015) The L3 syntax-discourse interface. Bilingualism: Language and Cognition, 18(2), 208-226.

Westergaard M (2009) Usage-based vs. rule-based learning: the acquisition of word order in wh-questions in English and Norwegian. Journal of Child Language, 36(5), 1023-1051.

Westergaard M, Mitrofanova N, Mykhaylyk R and Rodina Y (this issue) Crosslinguistic influence in the acquisition of a third language: The Linguistic Proximity Model. International Journal of Bilingualism. 